News \& Views for Zhang et al. NCHEM- 17010021-T

Stefan Howorka

DNA NANOTECHNOLOGY

\title{
Bringing lipid bilayers into shape
}

Lipid bilayers form the thin and floppy membranes that define the boundary of compartments such as cells. Now, a method to control the shape and size of bilayers using DNA nanoscaffolds has been developed. Such designer materials advance synthetic biology and could find use in membrane research.

\section{Stefan Howorka}

Lipid bilayers play a crucial biological role by forming a dynamic boundary around cells and subcellular organelle units. Membranes are also used in biomedicine to coat bioimaging agents or drugs. In contrast to proteins or DNA, membranes lack a defined structure. Instead the semifluid membranes are composed of amphiphilic lipids that typically form layers, within which the lipid molecules are able to move freely. Although this non-covalent nature makes lipid membranes fragile, this is also a virtue - by being deformable they can also be adapted into various dynamic forms, as needed for a specific function.

In biology, the task of shaping and stabilizing membranes is carried out by the cytoskeleton, a soft scaffold composed of interconnected protein rods that are linked to the membrane. The cytoskeleton is, however, not easy to engineer given its complex architecture. The rods are made up of intricately folded polypeptides which makes it difficult to design predictable changes in structures. This lack of experimental control is a limiting factor in several areas of research such as in membrane biophysics and cell biology, but also in the creation of synthetic cell-like structures for biotechnology. Writing in Nature Chemistry, Chenxiang Lin and coworkers have now achieved an important step towards addressing this issue by developing simple-to-build scaffolds from DNA that impose shape on liposomes, yet in highly tunable fashion ${ }^{1}$.

DNA is ideal to construct nanoscale objects with a designed shape. In a technological 
development spanning the last two decades, the carrier of genetic information has been retooled as building material ${ }^{2}, 3$. Predictively folding and assembling DNA strands into new structures is easy as the DNA duplexes are held together by the well understood Watson-Crick base-pairs. Furthermore, dedicated software programs aid the rational design, while component strands are available from commercial sources at low cost. By taking advantage of these favorable factors, DNA nanotechnology can produce nanoscale architectures of almost any imaginable 2D or 3D shape with straight or curved edges.

Lin et al. have applied these advanced fabrication techniques to engineer DNA scaffolds that also template lipid bilayers ${ }^{1}$. Their approach was to first design nanocages that support spherical liposomes inside the hollow scaffold, and then to exploit the cages' modular design to expand the range of accessible membrane shapes (Fig. 1). Each cylinder-like nanocage was composed of two DNA rings that were separated by four pillars (Fig. 1a). The design was tunable as both the rings' diameter and the pillars' lengths could be varied. As additional feature, the rings' outer faces carried connector sites (Fig. 1a). These were designed to assemble multiple cages into oligomeric DNA tubes in the presence of connecting DNA strands (Fig. 1a).

After carrying out the design, the cages were readily fabricated by heating and cooling a mixture of DNA strands carrying pre-selected sequences. This led matching DNA segments to pair up and form the programmed architecture. The cages were then assembled into linear nanoscaffolds by adding the connecting strands (Fig. 1a). To position liposomes inside the cages, hydrophobic lipid anchors were attached to the cages' DNA rings at inward facing positions (Fig. 1a). Lipid anchoring has previously been used with other bilayer-floating DNA nanostructures ${ }^{4,5,6}$ and membrane-puncturing DNA nanopores ${ }^{7,8,9}$. In the work of Lin and coworkers, phospholipid anchors were tethered to the DNA rings. Afterwards, an excess of free lipids was added so that liposomes could form via self-assembly.

The validity of the approach was first demonstrated with linear assemblies of cages with $49 \mathrm{~nm}$ inner diameter and an inter-ring spacing of $56 \mathrm{~nm}$ (Fig. 1a). Analysis by electron microcopy indeed visualized both DNA tubes and enclosed spherical liposomes. Following the successful proof-of-principle experiment, the cages' four pillars were shortened to position the rings closer together (Fig. 1b). Consequently, the lipid-modified rings were now near enough to template elongated membrane tubes rather than separate liposomes (Fig. 1c). Similarly, wider and narrower membrane tubes were obtained when the DNA rings' diameter was changed to 66 $\mathrm{nm}$ or $28 \mathrm{~nm}$, respectively. 
To further illustrate the power of the modular design, the pillars of the DNA cage were strategically shortened. This altered the cage's shape from a cylinder to a wedge (Fig. 1c). Once assembled, arrays of cages had the expected form of a donut (Fig. 1c). Strikingly, the circular DNA scaffold imposed the same shape onto the membranes even though donut-shaped bilayers have not been reported previously. When the cages' pillars were further asymmetrically shortened, the resulting scaffolds produced helical membrane tubes that resemble corkscrew-like bacteria, as well as stacks of membrane disks that are similar to intracellular organelles. Finally, membrane shape was dynamically controlled by reconfiguring the scaffold with trigger strands. In one experiment, addition of strands shortened pillars' length and fused spherical liposomes into the longer membrane tube (Fig. 1a,b). In a similar fashion, a straight scaffold with the internal linear membrane tube was bent into a curved shape by switching the component DNA cages from cylinder to wedge shape.

The work reported by Lin and co-workers stands out because their modularly designed DNA cages elegantly created higher-order nanostructures capable of templating many different bilayer shapes. Earlier reports relied on a ring-like DNA scaffold to control the diameter of the enclosed liposomes but these demonstrations were limited to spherical shapes ${ }^{10,11}$. In another report, a DNA nanoball has been coated with a membrane to create a virus-like particle ${ }^{12}$. But no previous study has provided such as general route to a wide range of dynamically changing membrane morphologies including tubes, rings, spirals, and 3D arrays.

How does the new approach compare to existing techniques that form bilayers? Clearly, using DNA scaffolds is experimentally more involved than simpler routes without templates. For example, vesicles of different diameters can be directly self-assembled but the resulting size distributions are much wider than in templated routes. Liposomes can also be formed by continuous flow in microfluidic network even though the resulting shapes are usually limited to spheres Other techniques can flatten vesicles within microfluidic chambers or deform them by pulling with microneedles; however, only a limited range of bilayer morphologies are accessible, and only a relatively low number of membrane copies is usually obtained. DNA scaffolds therefore complement existing membrane techniques because multiple shapes are accessible at relatively high copy numbers with only very minor variations in diameter. One drawback is that the hollow scaffold does not currently provide a route to fabricate asymmetric bilayers. In asymmetric membranes, the bilayers' two leaflets have different lipid compositions. Template-free emulsion methods allow the leaflets to be assembled one after the other thus enabling the formation of different compositions. In contrast, the approach of Lin and co- 
workers currently forms both sheets at the same time inside the scaffold.

Where can DNA templating be used and how can the technology be expanded? One of the first applications could lie in membrane biophysics. A key challenge is to understand how the morphology of the membrane influences its dynamics and composition, as well as the function of membrane proteins within biological systems such as for photosynthesis or oxidative respiration. The availability of easy-to-engineer scaffolds provides an avenue to advance research by enabling pre-determination of bilayer morphology so that its influence on bilayer dynamics and function can be probed. Similarly, within synthetic biology one can now envisage the construction of organelle-like compartments that replicate photosynthesis and other biological processes where folded membranes play an important role. Furthermore, an intriguing prospect would be to re-think Lin et al.'s modular DNA exo-skeleton approach that currently supports membranes from the outside. Turning the idea inside-out, an DNA endoskeleton ${ }^{12}$ would render the assemblies more cell-like, This approach might also make possible the rational construction of asymmetric membranes. Moreover, the assembly of ever larger DNA scaffolds would lead to synthetic membrane constructs resembling bacteria with a diameter of around 1 micrometer. In conclusion, templating membranes with DNA nanoscaffolds is a powerful route that has the potential to shape synthetic biology, membrane research, and biotechnology ${ }^{13}$.

Stefan Howorka is in the Department of Chemistry and the Institute of Structural and Molecular Biology, University College London, London, WC1H OAJ, UK. e-mail: s.howorka@ucl.ac.uk 


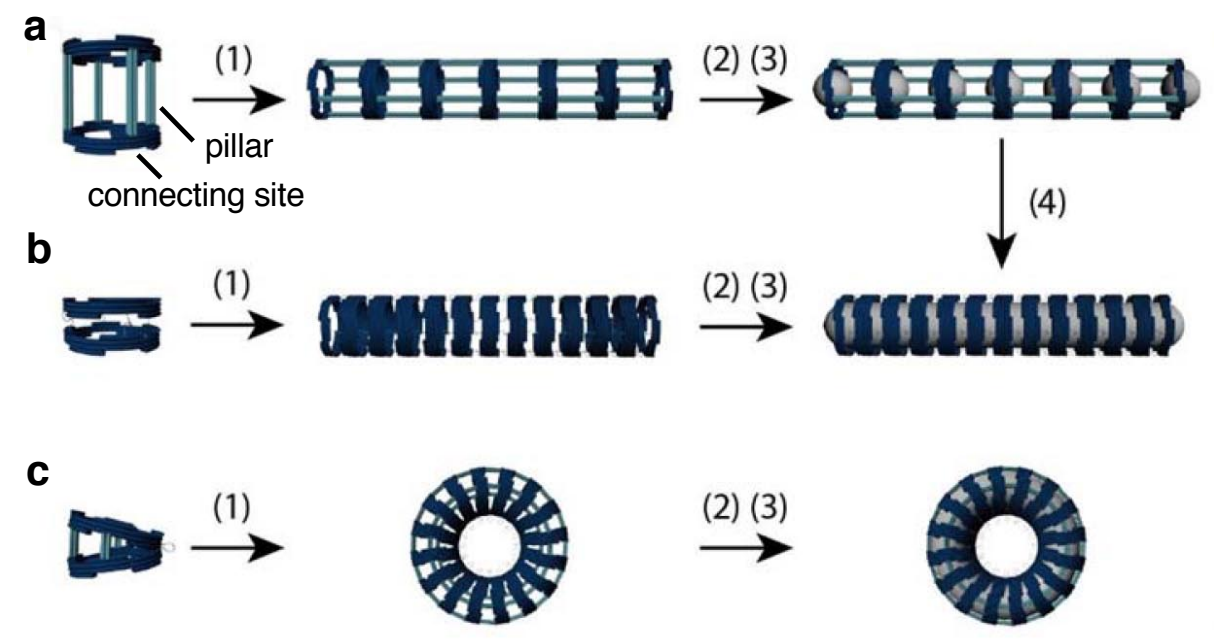

Figure 1. DNA nanoscaffolds template bilayer vesicles of tunable size and morphology. a) The scaffold's structural unit, a DNA nanocage, is composed of two rings and four pillars. Connector sites on the rings serve to assemble multiple cages. Adding connector strands (1) assembles nanocages into a multi-component scaffold. Subsequent addition of lipid-modified DNA (2) places membrane anchors to the scaffold. Mixing with free lipids followed by dialysis (3) leads to the formation of linear arrays of membrane vesicles. b) A DNA cage with single stranded, shortened pillars is the unit of another nanoscaffold where the lipid anchored rings are closer than in (a). The scaffold hence templates an elongated bilayer tube. The DNA-bilayer tube is also obtained by (4) adding a trigger strands that remove component strands of the pillars and thereby renders them single stranded and shortened. c) A nanocage with two shortened pillars resembles a wedge. The resulting multimeric DNA scaffold and membrane has a donut-like shape. Figure reproduced from reference 1.

1. Zhang Z., Yang Y., Pincet F., Llaguno M., Lin C. Nat. Chem. 9, x (2017).

2. Chen Y. J., Groves B., Muscat R. A., Seelig G. Nat. Nanotechnol. 10, 748-760 (2015).

3. Pinheiro A. V., Han D., Shih W. M., Yan H. Nat. Nanotechnol. 6, 763-772 (2011). 
4. Czogalla A., et al. Angew. Chem. Int. Ed. 54, 6501-6505 (2015).

5. Kocabey S., et al. ACS Nano 9, 3530-3539 (2015).

6. Johnson-Buck A., Jiang S., Yan H., Walter N. G. ACS Nano 8, 5641-5649 (2014).

7. Langecker M., et al. Science 338, 932-936 (2012).

8. Gopfrich K., et al. Nano Lett. 15, 3134-3138 (2015).

9. Burns J. R., Seifert A., Fertig N., Howorka S. Nat. Nanotechnol. 11, 152-156 (2016).

10. Yang Y., et al. Nat. Chem. 8, 476-483 (2016).

11. Xu W., et al. J. Am. Chem. Soc. 138, 4439-4447 (2016).

12. Perrault S. D., Shih W. M. ACS Nano 8, 5132-5140 (2014).

13. Howorka S. Science 352, 890-891 (2016). 